\title{
ERRATUM
}

\section{Erratum zu: Rohstoffwirtschaft und gesellschaftliche Entwicklung}

\author{
Peter Kausch • Jörg Matschullat•Martin Bertau • Helmut Mischo
}

(C) Springer-Verlag Berlin Heidelberg 2016

P. Kausch et al., (Hrsg.), Rohstoffwirtschaft und gesellschaftliche Entwicklung, DOI 10.1007/978-3-662-48855-3

\section{Erratum zum Titel: Peter Kausch • Jörg Matschullat • Martin Bertau Helmut Mischo (Hrsg.) Rohstoffwirtschaft und gesellschaftliche Entwicklung 10.1007/978-3-662-48855-3}

Die Autoren sind in das Autorverzeichnis eingefügt und die Reihenfolge ist geändert.

Die aktualisierte Originalversion des Buches kann hier abgerufen werden $10.1007 / 978-3-662-48855-3$

Peter Kausch

TU Bergakademie Freiberg

Freiberg, Deutschland

Jörg Matschullat

Interdisziplinäres Ökologisches Zentrum

TU Bergakademie Freiberg

Freiberg, Deutschland

Martin Bertau

TU Bergakademie Freiberg

Freiberg, Deutschland

Helmut Mischo

TU Bergakademie Freiberg

Freiberg, Deutschland 